\title{
Generation of White-Light Optical Vortices through Cascaded Four-Wave Mixing
}

\author{
P. Hansinger ${ }^{1}$, G. Maleshkov ${ }^{2}$, I. Garanovich ${ }^{3}$, D. Skryabin ${ }^{4}$, D. N. Neshev ${ }^{3}$, \\ Yu. S. Kivshar ${ }^{3}$, A. Dreischuh ${ }^{2}$, G. G. Paulus ${ }^{1}$ \\ ${ }^{1}$ Institute of Optics and Quantum Electronics, Friedrich-Schiller-University, Jena, Germany \\ ${ }^{2}$ Department of Quantum Electronics, Faculty of Physics, Sofia University, Sofia, Bulgaria \\ ${ }^{3}$ Nonlinear Physics Center, Research School of Physics and Engineering, Australian National University, Canberra ACT 0200, Australia \\ ${ }^{4}$ Centre for Photonics and Photonic Materials, University of Bath, Bath, United Kingdom \\ Dragomir.Neshev@anu.edu.au
}

\begin{abstract}
We study numerically and experimentally the process of cascaded four-wave mixing of a vortex beam imposed on short laser pulses propagating in a gas cell. We demonstrate that such cascaded nonlinear process leads to the generation of white-light optical vortices with well preserved vortex phase profile.
\end{abstract}

\section{Introduction}

Optical vortices (OVs) are singular optical beams with a spiral phase dislocation in their wavefront. When two pump beams at frequencies $\omega_{1}$ and $\omega_{2}$ interact in a Kerr-type nonlinear medium, the four-wave mixing (FWM) process results in the generation of new distinct sum and difference frequency components at the output. Importantly, unlike OV propagation in a Raman [1] or quadratic nonlinear medium [2] the FWM process is expected to preserve the topological charge of the $O V S$, and thus it can be employed for the generation of white-light vortices. However, as the FWM process is accompanied by noticeable nonlinear instabilities due to self- and cross-phase modulation (SPM and (PM), the phase information in the newly generated frequency components may be destroyed [3]. Therefore, it is important to reveal the possible regimes where the FWM process will dominate nonlinear instabilities, leading to the stable generation of white-light optical vortices.

In this work, we report on the first study of the non-phase-matched FWM of type $\omega_{\mathrm{s}}=\omega_{1}+\omega_{2}-\omega_{3}$ involving pump beams carrying two-dimensional spatial phase dislocations in the form of single-charged OVs. We demonstrate that by careful choice of the parameters of the filamentation process, a white-light vortex beam is generated in the process of supercontinuum generation.

\section{Numerical studies}

Our model is based on a set of 10 coupled nonlinear partial differential Schrödinger-type equations for the equallyspaced frequency components $\omega_{\mathrm{j}}(\mathrm{j}=1, \ldots, 10)$. The model takes into account the two-dimensional beam diffraction, FWM effect, as well as SPM and CPM effects. While the analytical form of the model with ten or more frequency components is quite cumbersome due to the large number of cross nonlinear terms, an idea for involved nonlinear terms can be obtained in the gradually simpler case of 4 involved frequency components ( 2 pump waves at frequencies $\omega$ and $\omega+\delta$ and 2 signal waves at frequencies $\omega-\delta$ and $\omega+2 \delta$ ). In this case the model equations read:

$$
i \frac{\partial A_{m}}{\partial z}+L_{D i f f}^{\omega} k_{m} A_{m}+\frac{\lambda_{m} n\left(\lambda_{\omega}\right)}{2 \lambda_{\omega} n\left(\lambda_{m}\right)} \Delta_{\perp} A_{m}+\gamma\left\{\left|A_{m}\right|^{2} A_{m}+2 \sum_{n \neq m}\left|A_{n}\right|^{2} A_{m}+H_{m}\right\}=0,
$$

( $m, n=\omega-\delta, \omega, \omega+\delta, \omega+2 \delta$ ). Here the terms in the brackets account for the SPM and for the CPM,

$$
\begin{aligned}
& H_{\omega-\delta}=2 A_{\omega+2 \delta}^{*} A_{\omega} A_{\omega+\delta} \exp \left(i \Delta k_{1} z L_{D i f f}^{\omega}\right)+A_{\omega}^{2} A_{\omega+\delta}^{*} \exp \left(i \Delta k_{2} z L_{D i f f}^{\omega}\right), \\
& H_{\omega}=2 A_{\omega}^{*} A_{\omega+\delta} A_{\omega-\delta} \exp \left(i \Delta k_{3} z L_{D i f f}^{\omega}\right)+2 A_{\omega+\delta}^{*} A_{\omega+2 \delta} A_{\omega-\delta} \exp \left(i \Delta k_{4} z L_{D i f f}^{\omega}\right)+A_{\omega+\delta}^{2} A_{\omega+2 \delta}^{*} \exp \left(i \Delta k_{5} z L_{D i f f}^{\omega}\right), \\
& H_{\omega+\delta}=2 A_{\omega} A_{\omega+2 \delta} A_{\omega-\delta} \exp \left(i \Delta k_{6} z L_{D i f f}^{\omega}\right)+2 A_{\omega+\delta}^{*} A_{\omega+2 \delta} A_{\omega} \exp \left(i \Delta k_{7} z L_{D i f f}^{\omega}\right)+A_{\omega}^{2} A_{\omega-\delta}^{*} \exp \left(i \Delta k_{8} z L_{D i f f}^{\omega}\right), \\
& H_{\omega+2 \delta}=2 A_{\omega-\delta}^{*} A_{\omega} A_{\omega+\delta} \exp \left(i \Delta k_{9} z L_{D i f f}^{\omega}\right)+A_{\omega+\delta}^{2} A_{\omega}^{*} \exp \left(i \Delta k_{10} z L_{D i f f}^{\omega}\right),
\end{aligned}
$$

reflect the different possible FWM processes, which depend on the respective phase matching

$$
\begin{array}{ll}
\Delta k_{1}=k_{\omega}+k_{\omega+\delta}-2 k_{\omega+2 \delta}-k_{\omega-\delta} & \Delta k_{6}=k_{\omega+2 \delta}+k_{\omega-\delta}-k_{\omega}-k_{\omega+\delta} \\
\Delta k_{2}=2 k_{\omega}-k_{\omega+\delta}-k_{\omega-\delta} & \Delta k_{7}=k_{\omega+2 \delta}+k_{\omega}-2 k_{\omega+\delta} \\
\Delta k_{3}=k_{\omega+\delta}+k_{\omega-\delta}-2 k_{\omega} & \Delta k_{8}=2 k_{\omega}-k_{\omega-\delta}-k_{\omega+\delta} \\
\Delta k_{4}=k_{\omega+2 \delta}+k_{\omega-\delta}-k_{\omega+\delta}-k_{\omega} & \Delta k_{9}=k_{\omega}+k_{\omega+\delta}-k_{\omega-\delta}-k_{\omega+2 \delta} \\
\Delta k_{5}=2 k_{\omega+\delta}-k_{\omega+2 \delta}-k_{\omega} & \Delta k_{10}=2 k_{\omega+\delta}-k_{\omega}-k_{\omega+2 \delta},
\end{array}
$$


where $k_{m}$ stands for the wavenumber of the $m$-th wave inside the nonlinear medium (NLM). Since we assume that initially the two high-intensity pump waves are at frequencies $\omega$ and $\omega+\delta$, it is natural to express the propagation coordinate $x$ in units of diffraction lengths of one of these waves. We choose normalization to the Rayleigh diffraction length of the optical vortex at frequency $\omega$, i.e. to $L_{D i f f}^{\omega}=k_{\omega} a^{2}$, where $a$ is the radius of the OV beam, being the same for both pump waves. The linear term in Eq. (1) comprising $\Delta_{\perp}$ accounts for the two-dimensional beam diffraction, whereas $\gamma$ is the Kerr nonlinear coefficient. $\operatorname{sign}(\gamma)$ is positive, reflecting our experimental condition. The two pump waves are chosen to have central wavelengths of $780 \mathrm{~nm}$ and $800 \mathrm{~nm}$. All necessary refractive indices and wave numbers are calculated at these pump wavelengths. The input pump OV beams are described by

$$
A=A_{0} B(r) \tanh \left(r / r_{0}\right) \exp (i l \varphi),
$$

where $A_{0}$ is the peak field amplitude, $B(r)$ is the Gaussian beam carrying the optical vortex, $B(r)=\exp \left(-r^{2} / a^{2}\right)$, $\tanh \left(r / r_{0}\right)$ describes the OV core, and the integer $l$ is the OV topological charge ( $l= \pm 1$ throughout this work). The background beam width $a$ is chosen sufficiently broader than the vortex core.

In the complete 10-wave-model, the two pump beams have frequencies located in the center of the spectrum, $\omega_{\mathrm{p}}$ $(\mathrm{p}=5$ and $\mathrm{p}=6)$. When both pump OV beams carry unit topological charges of the same sign, our numerical simulations predict generation of signal beams $\omega_{\mathrm{s}}(\mathrm{s}=1 . .4$ and $\mathrm{s}=7 . .10)$ all carrying OVs of the same charge. This is shown in the examples for $s=6$ to 10 in Fig. 1. While the beam profile is slightly different for each spectral component, the phase indicates the presence of a well defined vortex of charge 1 . These results confirm that the generation of ultra-broad spectrum vortex beams is possible through cascaded FWM process. The condition is that the SPM and CPM are sufficiently weak to reduce the possible vortex azimuthal instabilities.
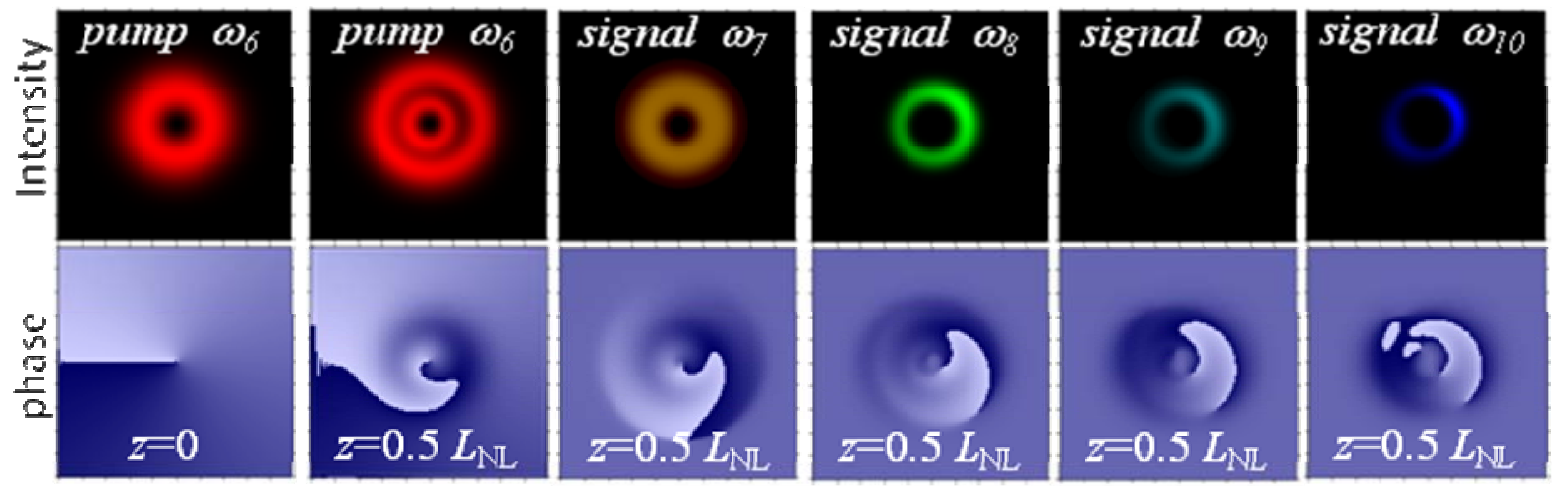

Fig 1 Numerical results. Intensity (upper row) and phase (lower row) of one of the pump waves at the entrance ( $z=0)$ and at the exit of the nonlinear medium $\left(z=0.5 L_{\mathrm{NL}}\right)$. Left two columns show the pump beam at the input and at the output of the cell. For singly-charged pump waves the generated lower-frequency components (not shown here) are symmetric to the higher-frequency ones with respect to the pump waves.

In our simulations we have also tested the case of different charges of the pump beams. If the two pump beams at frequencies $\omega_{5}$ and $\omega_{6}$ carry OVs with opposite unit charges, the generated signals at frequencies $\omega_{4}$ and $\omega_{7}$ carry charge-three vortices which, eventually, decay into three charge-one vortices with highly overlapping cores. When the input pump beams contain a charge-one OV and a fundamental Gaussian beam, generation of opposite chargetwo and charge-one vortices is predicted in the restricted 4-wave model. In the complete 10-wave model, due to the cascaded nature of the FWM and due to the shifts of the vortex cores, we observed even richer dynamics of vortices.

While our model neglects the full spatiotemporal dependence in the filamentation process as well as the influence of the generated plasma in the gas cell, it accurately captures the spectral reshaping of the output beam. However, more sophisticated models are required to fully capture the inherently complex dynamics of the process. This is especially important for the case of higher-charge vortices, which are much more prone to instabilities.

\section{Experimental results}

To verify these predictions we conducted experiments in an Ar-filled gas cell with Brewster windows kept under adjustable pressure of 0.8-2.3 bar. The experimental setup is shown in Fig. 2. As a pump beam we use $1 \mathrm{kHz}$ train of amplified 30-fs-pulses at $\lambda_{\mathrm{c}}=795 \mathrm{~nm}$ from a multi-pass Ti:Sapphire amplifier, delivering in the NLM 50fs pulses of up to $2.1 \mathrm{~W}$ average power. The vortex beam profile is generated via a spiral phase plate imprinted in a fused silica substrate. The beam is focused into the gas cell with a $2 \mathrm{~m}$ curved mirror. The output of the cell is imaged onto a camera as well as the spectrum is measured onto a CCD spectrometer. A typical input and output spectrum of the 
cell is shown in Fig. 2-right. Clearly, we observe the generation of broad supercontinuum output beam with frequency range spanning from approximately $650 \mathrm{~nm}$ to more than $950 \mathrm{~nm}$.

Importantly, the input spectrum of the pulses can be switched to either Gaussian or modulated (two peaks) to mimic simulation conditions. The peak intensity and gas pressure are carefully controlled in order to vary the strength of nonlinearity. Higher nonlinearity results in stronger spectral broadening, however it also increases the vortex instability (beam breakup) due to the SPM and CPM effects. An advantage of the used gas cell in comparison to previously used $\mathrm{CaF}_{2}$ samples [3] is the low dispersion and the good phase-matching conditions for FWM.
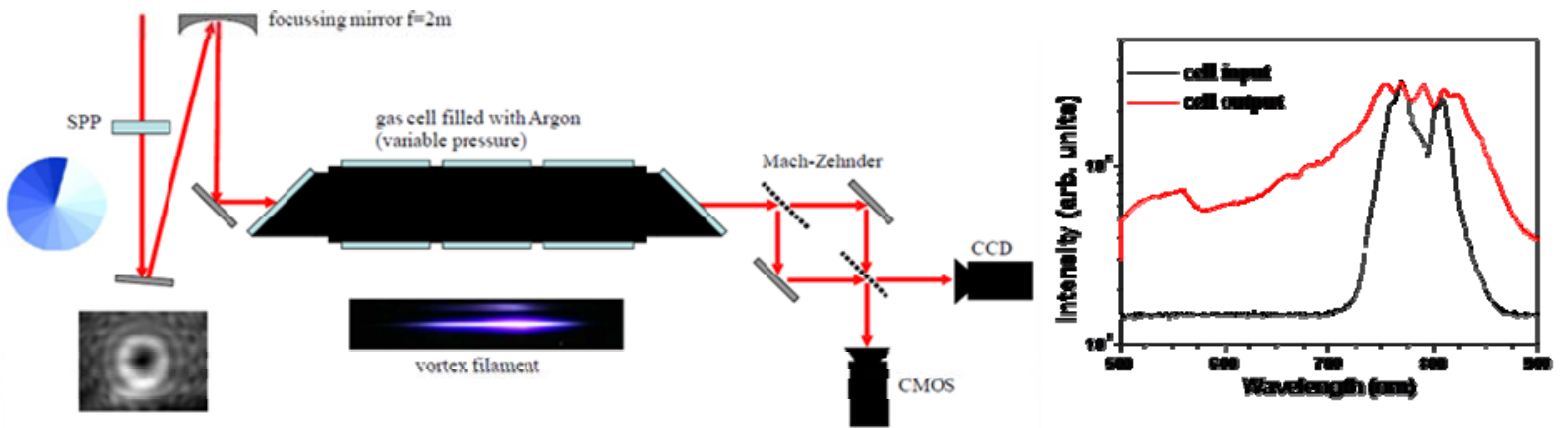

Fig 2 Experimental setup: Vortex phase is imprinted on a Gaussian beam with a 16-level spiral phase plate (fused silica). Gas cell: length 1.5m with Brewster windows filled with Argon at pressure $\mathrm{p}=0.8-2.3$ bar. Right: typical input and output spectrum of the pulses.

To test the phase structure of the different spectral components of the continuum, we also perform interferometric measurements in every $40-\mathrm{nm}$-wide spectral window within this interval. Four typical measurements of the interfering of the output beam with itself are shown in Fig. 3. These measurements confirm that all spectral components in the generated continuum indeed carry single-charged OVs, leading to the nonlinear generation of white-light vortex beams.
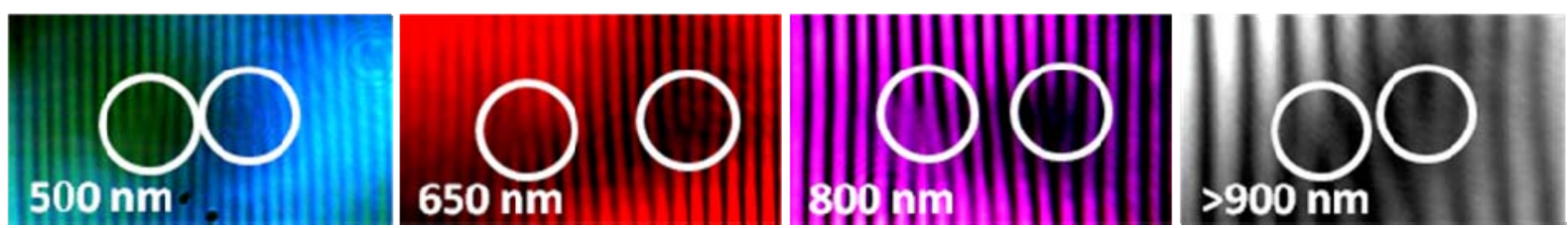

Fig 3 Experimental results. Self interference of portions of the generated white light pre-selected by interference filters with a transmission bandwidth of $40 \mathrm{~nm}$, indicating the existence of singly-charged optical vortices in the pump (at $800 \mathrm{~nm}$ ) and in the newly generated spectral components. The respective central wavelengths are denoted.

\section{Conclusions}

Topological charge transfer of vortex beams to white-light supercontinuum vortex beam trough nonlinear frequency conversion has been predicted and experimentally demonstrated. While notable instabilities of the vortex beam occur through the beam propagation, the conservation of the net TC follows the predicted scheme for vortices with unit charge. Our results open new possibilities generation of vortex beam of arbitrary spectral profiles for applications in STED microscopy and super-resolution nanofabrication. Further studies are aimed to reveal the strong spatio-temporal coupling in the filamentation process for generation of complex spatiotemporal signals to test chemical reactions.

\section{References}

[1] A. V. Gorbach and D. V. Skryabin, "Cascaded generation of multiply charged optical vortices and spatiotemporal helical beams in a Raman medium,” Phys. Rev. Lett. 98, 243601 (2007).

[2] Berzanskis, A., Matijosius, A., Piskarskas, A., Smilgevicius, V., Stabinis, A., "Conversion of topological charge of optical vortices in a parametric frequency converter," Opt. Commun. 140, 273-276 (1997).

[3] D. N. Neshev, A. Dreischuh, G. Maleshkov, M. Samoc, and Yu.S. Kivshar, "Supercontinuum generation with optical vortices," Opt. Express. 18, $18368(2010)$. 